\title{
The Risk Factors of Nonalcoholic Fatty Liver Disease in Morbidly Obese Patients Undergoing Bariatric Surgery in Iran
}

\author{
Ladan Aghakhani $\mathbb{D}^{1},{ }^{1}$ Neda Haghighat $\mathbb{D}^{2},{ }^{2}$ Masoud Amini $\mathbb{D}^{3},{ }^{3}$ Seyed Vahid Hosseini $\mathbb{D}^{4}$, \\ and Seyed Jalil Masoumi iD $^{5}$ \\ ${ }^{1}$ Student Research Committee, School of Nutrition and Food Sciences, Shiraz University of Medical Sciences, Shiraz, Iran \\ ${ }^{2}$ Laparoscopy Research Center, School of Medicine, Shiraz University of Medical Sciences, Shiraz, Iran \\ ${ }^{3}$ Laparoscopy Research Center, Department of Bariatric Surgery, Shiraz University of Medical Sciences, Shiraz, Iran \\ ${ }^{4}$ Department of Surgery, Colorectal Research Center, Shiraz University of Medical Sciences, Shiraz, Iran \\ ${ }^{5}$ Nutrition Research Center, School of Nutrition and Food Sciences, Shiraz University of Medical Sciences, Shiraz, Iran
}

Correspondence should be addressed to Seyed Jalil Masoumi; masoumi7415@gmail.com

Received 2 November 2021; Revised 16 December 2021; Accepted 27 January 2022; Published 7 February 2022

Academic Editor: Fariborz Mansour-Ghanaei

Copyright (c) 2022 Ladan Aghakhani et al. This is an open access article distributed under the Creative Commons Attribution License, which permits unrestricted use, distribution, and reproduction in any medium, provided the original work is properly cited.

\begin{abstract}
Background and Aims. Nonalcoholic fatty liver disease (NAFLD) is common in severely obese individuals undergoing bariatric surgery. Assessing the prevalence and severity of NAFLD seems crucial since it may affect the prevention or development of more severe forms of fatty liver. Methods. This cross-sectional study was conducted on 228 severely obese individuals undergoing bariatric surgery. Abdominal ultrasonography was done, and clinical and biochemical factors (liver enzymes, lipid profile, and fasting blood sugar (FBS)) were assessed. Results. The mean body mass index (BMI) was $43.45 \pm 5.92 \mathrm{~kg} / \mathrm{m}^{2}$. The prevalence of NAFLD was $49.12 \%$ (mild steatosis: $37.5 \%$, moderate steatosis: $36.6 \%$, and severe steatosis: $25.8 \%$ ). The main risk factors of NAFLD were weight $(p=0.002)$, BMI $(p=0.003)$, alanine aminotransferase (ALT) $(p<0.001)$, aspartate aminotransferase (AST) $(p<0.001)$, serum triglycerides (TGs) $(p=0.004)$, and FBS $(p=0.039)$. The results revealed a statistically significant decrease in the mean level of high-density lipoprotein cholesterol (HDL-C) $(p=0.044)$. However, no significant association was found between the severity of liver steatosis and the presence of comorbidities such as hypertension, diabetes, hypothyroidism, and dyslipidemia. Conclusions. More severe NAFLD was associated with increased weight and BMI. Elevated ALT, AST, TG, and FBS levels and decreased HDL-C levels were also the risk factors of NAFLD and its progress to more severe conditions.
\end{abstract}

\section{Introduction}

Nonalcoholic fatty liver disease (NAFLD) refers to excessive liver adiposity with various histological abnormalities, which can be classified into two categories: nonalcoholic fatty liver and nonalcoholic steatohepatitis (NASH). Nonalcoholic fatty liver has been defined as hepatic steatosis without any significant inflammation leading to hepatocellular injury or fibrosis, whereas NASH has been described as hepatic steatosis with inflammation. Given that NASH is a more severe stage of NAFLD, it is more likely to develop advanced cirrhosis, liver failure, and hepatocellular carcinoma $[1,2]$. The prevalence of NAFLD increases pri- marily by the increasing prevalence of obesity, diabetes mellitus (DM), hyperlipidemia, and polycystic ovary syndrome (PCOS) [3]. The global prevalence rate of NAFLD has been estimated as $25 \%$ in the general population [4]. In Iran, as a developing country, the prevalence of NAFLD has been reported as $21.5 \%$ [3]. Insulin resistance and dyslipidemia have been considered the main pathognomonic factors of the pathogenesis of NAFLD. Moreover, obesity and central obesity have been found to be associated with an increase in free fatty acid supply to the liver followed by insulin resistance [5].

Evidence of hepatic steatosis must be present on imaging or histology to diagnose NAFLD, and other causes of liver 
disease or steatosis must be excluded. In spite of the fact that liver biopsy is the gold standard for diagnosing and staging NAFLD, there are some limitations such as invasiveness and variability of sampling, causing this method not to be recommended for all patients. In this context, evaluation of hepatic steatosis by abdominal ultrasound has received much attention, because it is simple, inexpensive, easily tolerated, and noninvasive. Furthermore, it can provide valuable information. Generally, hepatic steatosis on abdominal ultrasound can be defined by at least two of the following findings: liver brightness, vascular blurring, increased hepatorenal contrast, and deep attenuation [6-8]. However, considering the increased prevalence of NAFLD when combined with morbid obesity, it is essential to recognize and evaluate the related risk factors and predictors. Therefore, the present study is aimed at estimating the prevalence of NAFLD and determining its most critical risk factors in morbidly obese patients undergoing bariatric surgery.

\section{Materials and Methods}

2.1. Patients and Study Design. This cross-sectional study was conducted on 228 patients with severe obesity undergoing bariatric surgery in the Obesity Center of Mother and Child Hospital in Shiraz between June 2020 and June 2021. We calculated the sample size using the prevalence of NAFLD in Asian countries (12-24\%) [9] with a power of $80 \%$. The primary outcome of the study was the prevalence of NAFLD, and the secondary outcome was the investigation of the clinical and biochemical risk factors of NAFLD among morbidly obese patients. The study was approved by the Institutional Ethics Committee of Shiraz University of Medical Sciences (IR.SUMS.REC.1399.245). After providing the patients with explanations about the purpose of the study and the confidentiality of their data, their written informed consent was obtained. The inclusion criteria were age $>18$ years and body mass index $(\mathrm{BMI}) \geq 40 \mathrm{~kg} / \mathrm{m}^{2}$ or $\mathrm{BMI} \geq 35$ $\mathrm{kg} / \mathrm{m}^{2}$ with one or more comorbidities attributable to obesity. The exclusion criteria were alcohol consumption $\geq 20 \mathrm{~g}$ per day for females and $\geq 30 \mathrm{~g}$ per day for males, menopause, lactation, hepatotoxic drugs intake, chronic hepatitis $\mathrm{B}$ or $\mathrm{C}$ virus infection, and other liver diseases.

2.2. Diagnosis of NAFLD. Abdominal ultrasound was used as the reference for diagnosing NAFLD among the patients. The same radiologist performed all ultrasound scans using a SonoAce R5 ultrasound device (SonoAce R5 ultrasound system, South Korea) with a convex probe of $3.5 \mathrm{MHz}$. According to the previously reported diagnostic criteria, ultrasonic hepatic steatosis was defined based on a comparative evaluation of image brightness compared to the kidneys. Fatty liver causes an increase in the echogenicity of the liver parenchyma [10]. Fatty liver grading has been described in Table 1 [11]. Accordingly, the grade of fatty liver was established.

\subsection{Data Collection}

2.3.1. Anthropometric Measurement. Weight was measured using a digital scale (Seca, Hamburg, Germany) to the near-
TABLE 1: Grading of nonalcoholic fatty liver on ultrasonography.

Mild liver steatosis (grade I)

(i) Least diffuse increase of liver echogenicity

(ii) The liver appears brighter than the kidney cortex

(iii) Standard visualization of intrahepatic vessel walls

Moderate liver steatosis (grade II)

(i) Moderate diffuse increase of liver echogenicity

(ii) A little defective visualization of the intrahepatic vessels

Severe liver steatosis (grade III)

(i) Severe increase of liver echogenicity

(ii) Poor or lack of visualization of intrahepatic vessels

(iii) Poor penetration of the posterior segment of the right lobe of the liver

TABLE 2: Demographic, anthropomorphic, and clinical data of the study population.

\begin{tabular}{|c|c|}
\hline No. of patients & 228 \\
\hline Age, year & $37.04 \pm 11.28$ \\
\hline Female/male & $187(80) / 41(18)$ \\
\hline Weight (kg) & $116.72 \pm 19$ \\
\hline Body mass index $\left(\mathrm{kg} / \mathrm{m}^{2}\right)$ & $43.45 \pm 5.92$ \\
\hline Waist circumference $(\mathrm{cm})$ & $126.47 \pm 14.03$ \\
\hline Waist-to-hip ratio & $0.94 \pm 0.09$ \\
\hline \multicolumn{2}{|l|}{ Obesity classification } \\
\hline Class 1 (body mass index: $30-34.9 \mathrm{~kg} / \mathrm{m}^{2}$ ) & $10(4.4)$ \\
\hline Class 2 (body mass index: $35-39.9 \mathrm{~kg} / \mathrm{m}^{2}$ ) & $50(22)$ \\
\hline Class 3 (body mass index: $40-49.9 \mathrm{~kg} / \mathrm{m}^{2}$ ) & $136(59.9)$ \\
\hline Super obesity (body mass index $>50 \mathrm{~kg} / \mathrm{m}^{2}$ ) & $31(13.7)$ \\
\hline \multicolumn{2}{|l|}{ Age at the beginning of obesity, no } \\
\hline Childhood (2-12 years) & $86(47.5)$ \\
\hline Teenage (13-17 years) & $20(11)$ \\
\hline Youth (18-39 years) & $67(37)$ \\
\hline Middle age ( $40-65$ years) & $7(3.9)$ \\
\hline Old age ( $>65$ years) & $1(0.6)$ \\
\hline Hypertension & $35(18.5)$ \\
\hline Type 2 diabetes mellitus & $17(7.8)$ \\
\hline Hypothyroidism & $38(21.1)$ \\
\hline Dyslipidemia & $78(36.1)$ \\
\hline
\end{tabular}

Waist circumference $(n=165)$, waist-to-hip ratio $(n=165)$, age at the beginning of obesity $(n=181)$, hypertension $(n=189)$, type 2 diabetes mellitus $(n=217)$, hypothyroidism $(n=180)$, and dyslipidemia $(n=216)$. The data have been presented as number (\%) or mean \pm standard deviation.

est $0.1 \mathrm{~kg}$ in light clothing and without shoes. Height was measured using a wall-mounted stadiometer to the nearest $0.1 \mathrm{~cm}$. BMI was calculated by dividing weight $(\mathrm{kg})$ by height squared (m). Hip circumference was measured at the level of the widest circumference portion of the buttocks. Waist circumference (WC) was also measured in the smallest part of the waist to the nearest $0.1 \mathrm{~cm}$. WC $>40$ inches $(102 \mathrm{~cm})$ among males and $>35$ inches $(88 \mathrm{~cm})$ among females was 
TABLE 3: Analysis of the clinical and biochemical risk factors of NAFLD.

\begin{tabular}{|c|c|c|c|c|c|}
\hline Risk factors & Grade I $(n=42)$ & $\begin{array}{c}\text { With NAFLD } \\
\text { Grade II }(n=41)\end{array}$ & Grade III $(n=29)$ & Without NAFLD $(n=116)$ & $p$ value \\
\hline Age, year & $37.14 \pm 12.56$ & $37.29 \pm 12.85$ & $37.68 \pm 10.34$ & $36.76 \pm 10.55$ & 0.980 \\
\hline \multicolumn{6}{|l|}{ Sex } \\
\hline Female & $39(92.9)$ & $31(75.6)$ & $19(65.5)$ & $98(84.5)$ & \multirow{2}{*}{0.018} \\
\hline Male & $3(7.1)$ & $10(24.4)$ & $10(34.5)$ & $18(15.5)$ & \\
\hline Weight $(\mathrm{kg})$ & $115.78 \pm 15.74$ & $121.69 \pm 19.68$ & $126.00 \pm 21.92$ & $112.99 \pm 18.11$ & 0.002 \\
\hline Body mass index $\left(\mathrm{kg} / \mathrm{m}^{2}\right)$ & $41.88 \pm 5.59$ & $46.64 \pm 5.27$ & $45.61 \pm 2.82$ & $42.22 \pm 5.87$ & 0.003 \\
\hline Waist circumference $(\mathrm{cm})$ & $122.90 \pm 9.82$ & $131.73 \pm 11.93$ & $132.80 \pm 12.07$ & $125.84 \pm 14.77$ & 0.392 \\
\hline Waist-to-hip ratio & $0.92 \pm 0.05$ & $0.94 \pm 0.05$ & $0.92 \pm 0.08$ & $0.94 \pm 0.11$ & 0.271 \\
\hline Hypertension $(n)$ & 4 & 10 & 5 & 16 & 0.065 \\
\hline Type 2 diabetes mellitus $(n)$ & 1 & 3 & 3 & 10 & 0.541 \\
\hline Hypothyroidism $(n)$ & 5 & 7 & 2 & 24 & 0.138 \\
\hline Dyslipidemia $(n)$ & 14 & 13 & 15 & 36 & 0.306 \\
\hline Alanine aminotransferase (IU/L) & $23.23 \pm 11.25^{\mathrm{a}, \mathrm{b}}$ & $40.48 \pm 28.64^{\mathrm{a}, \mathrm{c}}$ & $42.52 \pm 20.57^{\mathrm{b}, \mathrm{d}}$ & $25.40 \pm 15.00^{c, d}$ & $<0.001$ \\
\hline Aspartate aminotransferase (IU/L) & $20.76 \pm 7.07^{\mathrm{a}}$ & $28.76 \pm 13.98^{\mathrm{b}}$ & $32.52 \pm 14.88^{\mathrm{a}, \mathrm{c}}$ & $21.10 \pm 10.81^{\mathrm{a}, \mathrm{b}, \mathrm{c}}$ & $<0.001$ \\
\hline Alkaline phosphatase (IU/L) & $186.73 \pm 70.91$ & $196.40 \pm 51.04$ & $209.05 \pm 49.46$ & $190.73 \pm 60.41$ & 0.233 \\
\hline Total cholesterol (mg/dl) & $180.26 \pm 33.15^{\mathrm{a}}$ & $184.15 \pm 33.82^{\mathrm{b}}$ & $192.89 \pm 40.47^{\mathrm{a}, \mathrm{b}, \mathrm{c}}$ & $179.75 \pm 35.80^{\mathrm{c}}$ & 0.347 \\
\hline Triglyceride (mg/dl) & $137.88 \pm 55.40$ & $161.24 \pm 72.04$ & $191.52 \pm 95.62$ & $140.50 \pm 61.57$ & 0.004 \\
\hline Low-density lipoprotein (mg/dl) & $107.75 \pm 28.07$ & $116.52 \pm 28.65$ & $123.58 \pm 38.64$ & $109.10 \pm 29.87$ & 0.089 \\
\hline High-density lipoprotein (mg/dl) & $46.50 \pm 13.01$ & $39.98 \pm 11.46$ & $37.05 \pm 12.72$ & $44.13 \pm 10.89$ & 0.044 \\
\hline Fasting blood sugar (mg/dl) & $96.53 \pm 14.59^{\mathrm{a}}$ & $110.48 \pm 42.17$ & $106.35 \pm 16.55^{\mathrm{a}}$ & $100.59 \pm 26.30$ & 0.039 \\
\hline
\end{tabular}

Waist circumference $(n=165)$, waist to hip $(n=165)$, age at the beginning of obesity $(n=181)$, alanine aminotransferase $(n=218)$, aspartate aminotransferase $(n=218)$, alkaline phosphatase $(n=194)$, total cholesterol $(n=211)$, triglycerides $(n=213)$, low-density lipoprotein $(n=206)$, high-density lipoprotein $(n=183)$, and fasting blood sugar $(n=210)$. The data have been presented as mean \pm standard deviation for quantitative variables and number $(\%)$ for qualitative ones. ${ }^{\text {a,b,c }}$ Significant differences between the groups by the Bonferroni test.

considered a risk factor for health problems [12]. Waist-tohip ratio (WHR) was calculated by dividing WC by hip circumference. WHR $>0.85$ in females and $>1.00$ in males indicated a health risk [12]. Finally, a standardized protocol was used to measure systolic and diastolic blood pressure.

2.3.2. Biochemical Measurement. Biochemical measurements included alanine aminotransferase (ALT), aspartate aminotransferase (AST), alkaline phosphatase, total cholesterol, triglyceride (TG), high-density lipoprotein cholesterol (HDL-C), low-density lipoprotein cholesterol (LDL-C), and fasting blood sugar (FBS) levels. Moreover, type 2 diabetes mellitus (T2DM) was diagnosed based on the fasting plasma glucose concentration $\geq 126 \mathrm{mg} / \mathrm{dl}$, positive history, and being under antidiabetic medications.

2.4. Statistical Analysis. The collected data were analyzed using the SPSS software, version 22. The data were presented as mean \pm standard deviation (SD) or as absolute values and percentages. Kolmogorov-Smirnov test was used to analyze the normal distribution of the study variables. Then, chisquare test or Fisher's exact test was used to analyze the categorical variables. Univariate analysis of continuous variables was done using independent sample $t$-test and MannWhitney $U$ test (in case of nonnormal distribution of the variables). Moreover, changes in the clinical and biochemical variables were compared using the analysis of variance
(ANOVA) and Kruskal-Wallis nonparametric test, and Bonferroni test was used for pairwise comparisons. $p<$ 0.05 was considered statistically significant.

\section{Results}

3.1. Baseline Characteristics of the Patients Undergoing Bariatric Surgery. The anthropometric, demographic, and clinical characteristics of the enrolled patients have been presented in Table 2. The participants included 228 patients with the mean age of $37.04 \pm 11.28$ years. Among the patients, $187(80 \%)$ were female and $41(18 \%)$ were male. The mean weight of the participants was $116.72 \pm 19 \mathrm{~kg}$ (median: 112.20 and range: $80.40-180$ ), and their mean BMI was $43.45 \pm 5.92 \mathrm{~kg} / \mathrm{m}^{2}$ (median: 42.63 and range: $30.37-63)$. Moreover, 35 patients (18.5\%) had hypertension, 17 (7.8\%) had DM, 38 (21.1\%) had hypothyroidism, and 78 (36.1\%) had dyslipidemia.

3.2. Liver Steatosis. Overall, $49.12 \%$ of the participants had fatty liver. Mild liver steatosis (grade I) was found in 42 patients (37.5\%), moderate liver steatosis (grade II) in 41 patients (36.6\%), and severe liver steatosis (grade III) in 29 patients $(25.8 \%)$.

3.3. Risk Factors Associated with NAFLD. Comparison of the characteristics, comorbid conditions, and biochemical factors 


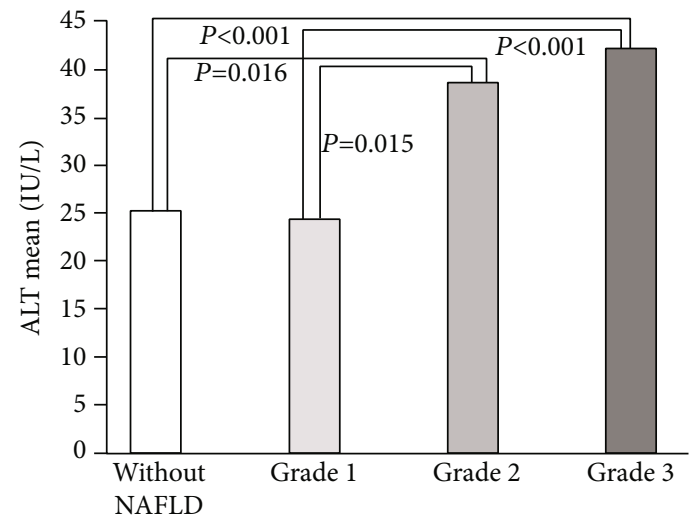

(a)

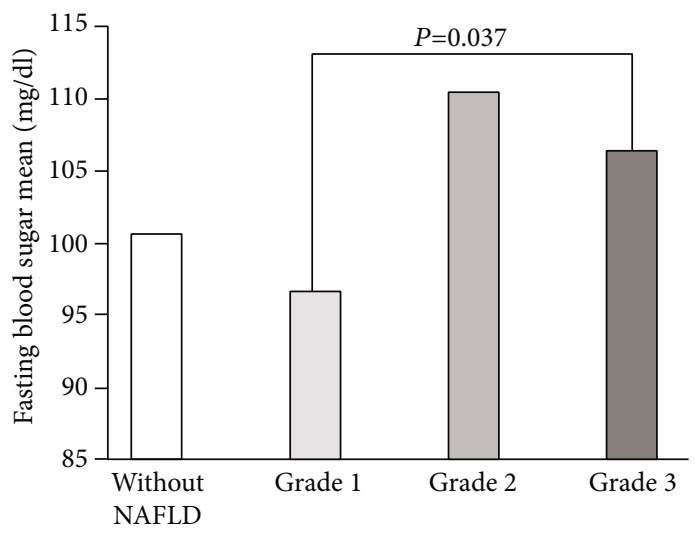

(c)

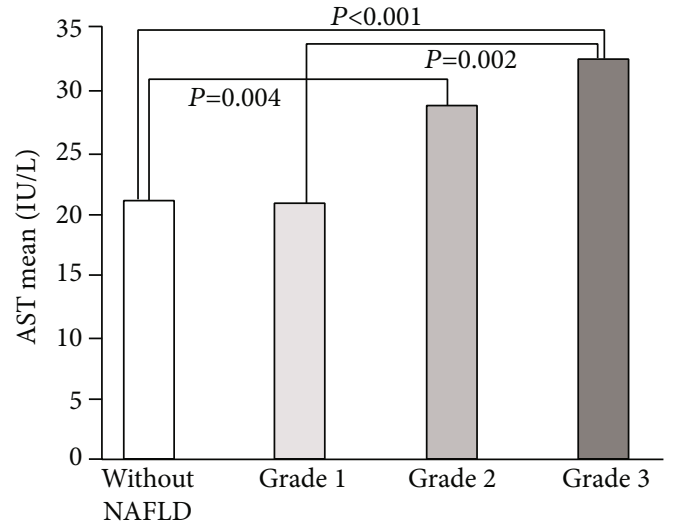

(b)

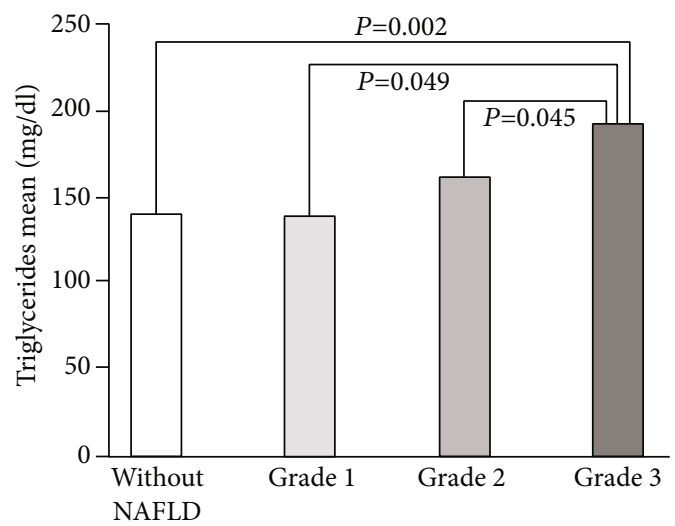

(d)

Figure 1: The means of (a) alanine aminotransferase (ALT), (b) aspartate transaminase (AST), (c) fasting blood sugar (FBS), and (d) triglycerides (TGs) in the patients with and without NAFLD undergoing bariatric surgery.

of the participants with and without NAFLD has been presented in Table 3. The results revealed no significant difference between the two groups in terms of age, WC, and WHR. As for weight and BMI, there was a significant difference between the patients with severe steatosis (grade III) and those without NAFLD $(p=0.005)$. The results also showed no statistically significant difference between the patients with and without NAFLD regarding comorbidities such as hypertension, diabetes, hypothyroidism, and dyslipidemia. Nonetheless, dyslipidemia was more prevalent in the NAFLD group (53.84\%).

Among the biochemical factors, those with statistically significant differences have been shown in Figure 1. Accordingly, serum ALT levels were higher in the patients with grade II $(40.48 \pm 28.64 \mathrm{IU} / \mathrm{L})$ and grade III $(42.52 \pm 20.57$ IU/L) liver steatosis compared to those with grade I liver steatosis $(23.23 \pm 11.25 \mathrm{IU} / \mathrm{L})$ and the group without NAFLD $(25.40 \pm 15 \mathrm{IU} / \mathrm{L})$. Serum AST levels were also higher in the patients with grade II $(28.76 \pm 13.98 \mathrm{IU} / \mathrm{L})$ and grade III $(32.52 \pm 14.88 \mathrm{IU} / \mathrm{L})$ in comparison to those without NAFLD $(21.10 \pm 10.81 \mathrm{IU} / \mathrm{L})$. There was also a statistically significant difference between the patients with grade I and grade III in this regard $(p=0.002)$. In addition, serum TG levels were higher in the patients with grade III (191.52 \pm $95.62 \mathrm{mg} / \mathrm{dl})$ than in those with grade II $(161.24 \pm 72.04$ $\mathrm{mg} / \mathrm{dl})(p=0.045)$, those with grade I $(137.88 \pm 55.40 \mathrm{mg} /$ dl) $(p=0.049)$, and the group without NAFLD (140.50 \pm $61.57 \mathrm{mg} / \mathrm{dl})(p=0.002)$. Fasting blood glucose was also higher in patients with grade III $(106.35 \pm 16.55 \mathrm{mg} / \mathrm{dl})$ compared to those with grade I $(96.53 \pm 14.59 \mathrm{mg} / \mathrm{dl})$ $(p=0.037)$. Furthermore, a significant decrease was observed in the mean level of HDL-C in the study population $(p=0.044)$.

\section{Discussion}

Obesity-associated NAFLD includes a spectrum of histological abnormalities ranging from steatosis to the inflammatory form of NAFLD, known as NASH. It is frequently seen in severe obesity, and its prevalence has been found to increase up to $90 \%$ in such patients $[13,14]$. However, little is known about the prevalence and severity of NAFLD among severely obese patients undergoing bariatric surgery in Iran. Therefore, the primary objective of this prospective cohort study was to identify the factors associated with the prevalence and severity of NAFLD in morbidly obese patients undergoing bariatric surgery.

The present study results indicated that the prevalence of NAFLD was $49.12 \%$, and the mean BMI of the patients was 
$43.45 \pm 5.92 \mathrm{~kg} / \mathrm{m}^{2}$. However, a lower prevalence (16.7\%) was reported in the study carried out by Karimi-Sari et al. [15]. On the other hand, some previous studies revealed a higher prevalence of NAFLD [16-18]. It should be noted that the majority of severely obese patients undergoing bariatric surgery are female.

The results of the current research showed no statistically significant association between NAFLD and the presence of comorbidities such as hypertension, diabetes, hypothyroidism, and dyslipidemia. Some previous studies also demonstrated no significant relationships between the increasing degrees of liver damage and hypertension, diabetes, and dyslipidemia [18-21]. However, some investigations have disclosed that diabetes was associated with NASH and advanced fibrosis $[20,22,23]$.

The current study findings indicated that the presence and severity of NAFLD were not significantly associated with age, WC, and WHR. However, increasing weight and BMI were significantly associated with NAFLD. Accordingly, patients with $\mathrm{BMI}>50 \mathrm{~kg} / \mathrm{m}^{2}(32.3 \%)$ were more likely to have severe NAFLD (grade III). Although several studies have emphasized the association between BMI and NAFLD/NASH $[16,19]$, most studies have shown no significant relationships between increasing BMI and NAFLD prevalence $[17,18,22]$.

In the current research, the severity of NAFLD was associated with increasing levels of ALT and AST. Among the patients, $23.1 \%$ had elevated levels of ALT ( $>41 \mathrm{IU} / \mathrm{L}$ in males and $>37 \mathrm{IU} / \mathrm{L}$ in females) and $8.3 \%$ had elevated levels of AST ( $>37 \mathrm{IU} / \mathrm{L})$. Moreover, the majority of patients with higher mean levels of liver enzymes had BMI $\geq 40 \mathrm{~kg} / \mathrm{m}^{2}$. It was previously established that metabolic syndrome and obesity were associated with elevated liver enzymes, particularly high serum ALT activity. Besides, almost $50 \%$ of obese people had elevated ALT levels in addition to NAFLD [24, 25]. In the same vein, Milić et al. conducted a large study on 799 obese patients and concluded that the median ALT and AST levels increased with the obesity class, exceeding the normal limits in $21 \%$ of the patients [26].

The present study results revealed a significant relationship between serum HDL-C, TG, and FBS levels and the occurrence of NAFLD, which was in agreement with the results of other studies conducted on the issue $[20,22,23]$. The relationship between obesity and hypertriglyceridemia is possibly mediated by insulin resistance, as studies have suggested that obesity raises the serum TG level as one of the acquired reasons. Moreover, insulin resistance has been recognized as one of the most important pathogeneses of NAFLD. In fact, obesity and hypertriglyceridemia are closely related to insulin resistance, leading to the development and progression of fatty liver [27].

The main limitation of the present study was doing the assessments using ultrasonography, while many previous studies made use of histology to diagnose NAFLD.

This was the first study that reported the status of NAFLD in severely obese Iranian patients undergoing bariatric surgery (sleeve gastrectomy, Roux-en-Y gastric bypass, and single anastomosis sleeve ileal) and analyzed the risk factors of NAFLD in this population.

\section{Conclusion}

In conclusion, the findings demonstrated that more severe NAFLD was associated with increasing weight and BMI, increasing levels of ALT, AST, TG, and FBS, and decreased HDL-C levels. However, hypertension, diabetes, hypothyroidism, and dyslipidemia were not associated with NAFLD and its severity. Detection and evaluation of NAFLD risk factors in severely obese patients undergoing bariatric surgery may effectively prevent NAFLD or its progress to more severe conditions.

\section{Data Availability}

The data used to support the findings of this study are available from the corresponding author upon request.

\section{Conflicts of Interest}

The authors declare no conflict of interests.

\section{Acknowledgments}

This study was extracted from an MSc thesis in Shiraz University of Medical Sciences. The authors would like to thank Ms. A. Keivanshekouh at the Research Consultation Center (RCC) of Shiraz University of Medical Sciences for improving the use of English in the manuscript.

\section{References}

[1] J. M. Jorge, A. Golas, N. Patel, J. P. Gonzalvo, and M. M. Murr, "Management of nonalcoholic fatty liver disease and the role of bariatric surgery: a brief review for surgeons," Surgery for Obesity and Related Diseases, vol. 16, no. 5, pp. 699-703, 2020.

[2] P. H. Sweet, T. Khoo, and S. Nguyen, "Nonalcoholic fatty liver disease," Primary Care, vol. 44, no. 4, pp. 599-607, 2017.

[3] F. Mansour-Ghanaei, F. Joukar, S. N. Mobaraki, S. Mavaddati, S. Hassanipour, and M. Sepehrimanesh, "Prevalence of nonalcoholic fatty liver disease in patients with diabetes mellitus, hyperlipidemia, obesity and polycystic ovary syndrome: a cross-sectional study in north of Iran," Diabetes and Metabolic Syndrome: Clinical Research and Reviews, vol. 13, no. 2, pp. 1591-1596, 2019.

[4] M. de Vries, J. Westerink, K. H. Kaasjager, and H. W. de Valk, "Prevalence of nonalcoholic fatty liver disease (NAFLD) in patients with type 1 diabetes mellitus: a systematic review and meta-analysis," The Journal of Clinical Endocrinology and Metabolism, vol. 105, no. 12, pp. 3842-3853, 2020.

[5] S. Hafeez and M. H. Ahmed, "Bariatric surgery as potential treatment for nonalcoholic fatty liver disease: a future treatment by choice or by chance?," Journal of Obesity, vol. 2013, Article ID 839275, 11 pages, 2013.

[6] E. Hashimoto, M. Taniai, and K. Tokushige, "Characteristics and diagnosis of NAFLD/NASH," Journal of Gastroenterology and Hepatology, vol. 28, Suppl 4, pp. 64-70, 2013.

[7] J. K. Dyson, Q. M. Anstee, and S. McPherson, "Non-alcoholic fatty liver disease: a practical approach to diagnosis and staging," Frontline Gastroenterology, vol. 5, no. 3, pp. 211-218, 2014. 
[8] L. Castera, V. Vilgrain, and P. Angulo, "Noninvasive evaluation of NAFLD," Nature Reviews. Gastroenterology \& Hepatology, vol. 10, no. 11, pp. 666-675, 2013.

[9] S. Ashtari, M. A. Pourhoseingholi, and M. R. Zali, "Non-alcohol fatty liver disease in Asia: prevention and planning," World Journal of Hepatology, vol. 7, no. 13, pp. 1788-1796, 2015.

[10] Y. C. Lin, H. M. Lo, and J. D. Chen, "Sonographic fatty liver, overweight and ischemic heart disease," World Journal of Gastroenterology, vol. 11, no. 31, pp. 4838-4842, 2005.

[11] D. U. Mahaling, M. M. Basavaraj, and A. J. Bika, "Comparison of lipid profile in different grades of non-alcoholic fatty liver disease diagnosed on ultrasound," Asian Pacific Journal of Tropical Biomedicine, vol. 3, no. 11, pp. 907-912, 2013.

[12] S. Sánchez-García, C. García-Peña, M. X. Duque-López, T. Juárez-Cedillo, A. R. Cortés-Núñez, and S. Reyes-Beaman, "Anthropometric measures and nutritional status in a healthy elderly population," BMC Public Health, vol. 7, no. 1, p. 2, 2007.

[13] S. A. Polyzos, J. Kountouras, and C. S. Mantzoros, "Obesity and nonalcoholic fatty liver disease: from pathophysiology to therapeutics," Metabolism, vol. 92, pp. 82-97, 2019.

[14] A. X. Holterman, G. Guzman, G. Fantuzzi et al., "Nonalcoholic fatty liver disease in severely obese adolescent and adult patients," Obesity, vol. 21, no. 3, pp. 591-597, 2013.

[15] H. Karimi-Sari, S. M. Mousavi-Naeini, M. Ramezani-Binabaj, S. Najafizadeh-Sari, M. H. Mir-Jalili, and F. Dolatimehr, "Prevalence of non-alcoholic fatty liver disease in morbidly obese patients undergoing sleeve bariatric surgery in Iran and association with other comorbid conditions," Jundishapur Journal of Chronic Disease Care, vol. 4, no. 2, article e25128, 2015.

[16] A. Atri, S. A. Jiwanmall, M. B. Nandyal et al., “The prevalence and predictors of non-alcoholic fatty liver disease in morbidly obese women - a cross-sectional study from southern India," European Endocrinology, vol. 16, no. 2, pp. 152-155, 2020.

[17] C. T. Frantzides, M. A. Carlson, R. E. Moore et al., "Effect of body mass index on nonalcoholic fatty liver disease in patients undergoing minimally invasive bariatric surgery," Journal of Gastrointestinal Surgery, vol. 8, no. 7, pp. 849-855, 2004.

[18] P. Praveenraj, R. M. Gomes, S. Kumar et al., "Prevalence and predictors of non-alcoholic fatty liver disease in morbidly obese south Indian patients undergoing bariatric surgery," Obesity Surgery, vol. 25, no. 11, pp. 2078-2087, 2015.

[19] M. Moretto, C. Kupski, C. C. Mottin et al., "Hepatic steatosis in patients undergoing bariatric surgery and its relationship to body mass index and co-morbidities," Obesity Surgery, vol. 13, no. 4, pp. 622-624, 2003.

[20] C. Boza, A. Riquelme, L. Ibañez et al., "Predictors of nonalcoholic steatohepatitis (NASH) in obese patients undergoing gastric bypass," Obesity Surgery, vol. 15, no. 8, pp. 11481153, 2005.

[21] L. Spaulding, T. Trainer, and D. Janiec, "Prevalence of nonalcoholic steatohepatitis in morbidly obese subjects undergoing gastric bypass," Obesity Surgery, vol. 13, no. 3, pp. 347349, 2003.

[22] J. B. Dixon, P. S. Bhathal, and P. E. O'Brien, "Nonalcoholic fatty liver disease: predictors of nonalcoholic steatohepatitis and liver fibrosis in the severely obese," Gastroenterology, vol. 121, no. 1, pp. 91-100, 2001.

[23] S. A. Xanthakos, T. M. Jenkins, D. E. Kleiner et al., "High prevalence of nonalcoholic fatty liver disease in adolescents under- going bariatric surgery," Gastroenterology, vol. 149, no. 3, pp. 623-634, 2015.

[24] R. Abdalgwad, M. F. Rafey, C. Murphy et al., "Changes in alanine aminotransferase in adults with severe and complicated obesity during a milk-based meal replacement programme," Nutrition \& Metabolism, vol. 17, no. 1, pp. 1-9, 2020.

[25] G. Iacobellis, A. Moschetta, R. Buzzetti, M. C. Ribaudo, M. G. Baroni, and F. Leonetti, "Aminotransferase activity in morbid and uncomplicated obesity: predictive role of fasting insulin," Nutrition, Metabolism, and Cardiovascular Diseases, vol. 17, no. 6, pp. 442-447, 2007.

[26] S. Milić, D. Lulić, and D. Štimac, "Non-alcoholic fatty liver disease and obesity: biochemical, metabolic and clinical presentations," World Journal of Gastroenterology, vol. 20, no. 28, pp. 9330-9337, 2014.

[27] J. Xing, X. Guan, Q. Zhang, S. Chen, S. Wu, and X. Sun, "Triglycerides mediate body mass index and nonalcoholic fatty liver disease: a population-based study," Obesity Facts, vol. 14, no. 2, pp. 190-196, 2021. 